\title{
Losartan: Reduces Ascites, but induces Cancer? Again Some Stupid Statements from PNAS- Proceedings of the National Academy of Science of USA!
}

\author{
Georgi Tchernev ${ }^{1,2^{*}}$ and Ivanka Temelkova ${ }^{1,2}$ \\ ${ }^{1}$ Department of Dermatology, Venereology and Dermatologic surgery, Medical Institute of Ministry of Interior [MVR]; Sofia, Bulgaria \\ ${ }^{2}$ Onkoderma- Clinic for Dermatology, Venereology and Dermatologic Surgery; Sofia, Bulgaria
}

Received: July 28, 2019; Accepted: July 30, 2019; Published: August 1, 2019

*Corresponding author: Prof. Dr. Georgi Tchernev, Department of Dermatology, Venereology and Dermatologic Surgery, Medical Institute of Ministry of Interior [MVR), General Skobelev 79, 1606 Sofia, Bulgaria, E-mail: georgi_tchernev@yahoo.de

\section{No Conflict of Interest, No Financial Disclosure!}

The main purpose of the analyzes carried out by the colleagues in an recently published paper in PNAS-USA [Proceedings of the National Academy of Science of the United States of America) is focused 1) on the one hand on the in vitro effect of Losartan in murine ovarian carcinoma model, demonstrating that the adjuvant effect of losartan should be definitely synergistic with regard to the parallel-induced / or ongoing chemotherapy, and on the other hand 2) in addition, that malignant ascites in retrospectively observed patient group seems to be also reduced [1].

The retrospective analyzes of ovarian cancer patients [and parallel therapy with ARB or ACE blockers), according to the colleagues, showed improved overall survival, compared to similar patients who were treated with another antihypertensive drug [1].

Given the current information that angiotensin receptor blockers are likely to have a possible pro-cancerogenic effect [2, 3] [although it is currently unclear whether this effect is due to the carcinogenic impurities or the generic itself), we believe that these data should be looked exclusively critically [1, 4, and 5] due to the following circumstances:

1) In the data presented by the colleagues, there is a complete lack of information as to whether and how many retrospective patients were treated prior to the onset of tumors with ARBs? This information is shared in the introduction section of the described article without being specified or detailed [1]. It is unclear whether AR- blockers have been assigned in the occasion of arterial hypertension [as described by the authors themselves in the introduction [1]) or is it subsequently appointed in order to reduce malignant ascites? This should be the most important / key point/moment in the article, and without its full clarification, the significance of the results remains a serious issue.
2) If patients with ovarian carcinoma were treated with ARblockers for arterial hypertension before the diagnosis of tumors themselves, their potential procancerogenic effect should also be inevitably discussed? As well as the overall treatment time prior to the diagnosis of ovarian cancer or the appearance of the first clinical symptoms.

3) It would also matter/ it won't be irrelevant whether the respective batch of medicaments [in this case containing Losartan/currently recalled Losartan) were manufactured by pharmaceutical companies that currently have withdrawn their products from the market due to contamination with carcinogens?

Without clarifying these important facts at this moment, it would be difficult to make definitive assessments for the possible positive effect of sartans on the survival of patient groups [within unclear originally defined retrospectively treated groups), or to provide other evaluations concerning the response of the extracellular matrix in the form of solid stress reduction, improved local perfusion, increased delivery of chemotherapeutics, losartan induced tumor hypoxia, improved chemotherapeutic efficacy, etc [1].

In the event of a positive response to one of the abovementioned points [1-3], the ARB blocker in question could be considered at the same time as a possible inducer of neoplastic process/ ovarian cancer [4].

\section{References}

1. Zhao Y, Cao J, Melamed A, Worley M, Gockley A, Jones D, et al., Losartan treatment enhances chemotherapy efficacy and reduces ascites in ovarian cancer models by normalizing the tumor stroma. Proc Natl Acad Sci U S A. 2019; 116(6):2210-2219. doi:10.1073/ pnas.1818357116

2. Tchernev G, Temelkova I. Drug-Induced Melanoma: Irbesartan Induced Cutaneous Melanoma! First Description in the World Literature! 
Open Access Maced J Med Sci. 2019; 7(1):114-116. doi:10.3889/ oamjms.2019.042

3. Tchernev G, Temelkova I. Irbesartan Induced Cutaneous Melanoma! Second Case in the Medical Literature! Open Access Maced J Med Sci. 2019; 7(1):121-123. doi: 10.3889/oamjms.2019.043

4. Tchernev G, Temelkova I. Valsartan Induced Melanoma?! First Description in Medical Literature! Open Access Maced J Med Sci. 2018; 6(12):2378-2380. doi: 10.3889/oamjms.2018.517
5. Tchernev G, Temelkova I. Additional 4 cases of valsartan/irbesartaninduced melanomas? J Biol Regul Homeost Agents. 2019; 33(3):911912. [Epub ahead of print]

6. Tchernev G, Temelkova I. Valsartan/hydrochlorothiazide induced prostate carcinoma in a patient who subsequently developed melanoma. J Biol Regul Homeost Agents. 2019; 33(4). [Epub ahead of print] 